\title{
Montage d'holographie numérique dynamique pour la nano-métrologie optique sans lentille
}

\author{
Maxime Jacquot ${ }^{1}$, Omar Iken ${ }^{1}$, Bastien Bobillier ${ }^{1}$, Sylvain Cremer $^{1}$, Luc Froehly ${ }^{1,2}$, François Courvoisier ${ }^{1,2}$, \\ Lucas Furfaro ${ }^{1}$, Remo Giust ${ }^{1}$, John Dudley ${ }^{1}$ \\ maxime.jacquot@univ-fcomte.fr
}

\author{
Adresse : ${ }^{1}$ Université de Franche-Comté, U.F.R. ST, Cursus Master en Ingénierie PICS \\ ${ }^{2}$ Centre National de la Recherche Scientifique, section 08 \\ FEMTO-ST / Dépt. d'Optique P.M. Duffieux UMR CNRS 6174 \\ 16 route de Gray, 25030 Besançon cedex, France
}

\begin{abstract}
RESUME : Ce travail concerne l'étude expérimentale d'un banc d'holographie numérique associé à un modulateur spatial de lumière (SLM). L'acquisition d'un hologramme sur un capteur 2D CMOS grâce à un interféromètre de type Michelson est présentée. L'objet est de taille millimétrique. La restitution en intensité et en phase de l'image est numérique. On montre ainsi que ce procédé autorise la mesure de différence de trajets optiques, sans recours à des lentilles, avec des résolutions pouvant atteindre la dizaine de nanomètres. L'insertion d'un élément adressable (SLM) dans le montage donne accès à une grande flexibilité à l'expérimentation pour deux raisons principales : des objets de phase pure modifiables sont générés grâce au SLM et une correction dynamique du front d'onde peut s'appliquer afin de compenser des aberrations du montage. Dans ce contexte, ce banc d'holographie numérique dynamique constitue un formidable outil pédagogique pour des étudiants en licence $3^{\text {ème }}$ année de Physique jusqu'à un niveau de master $2^{\text {ème }}$ année (CMI PICS). Cette étude repose sur des enseignements fortement pluridisciplinaires dispensés sous forme de $\mathrm{CM} / \mathrm{TD} / \mathrm{TP}$ intégrés mais aussi en projet d'étude et en stage de laboratoire, garantissant ainsi et ce dès la licence, un continuum entre la formation et la recherche.
\end{abstract}

Mots clés : dispositif pédagogique numérique et expérimental, holographie numérique dynamique, modulateur spatial de lumière, nano-métrologie optique.

\section{INTRODUCTION}

Cette étude provient d'un travail pédagogique qui débute en licence de physique $3^{\text {ème }}$ année et qui se poursuit jusqu'en master $2^{\text {ème }}$ année de sciences de la matière, spécialité PICS, de l'UFR-ST de l'Université de Franche-Comté [1]. Ce parcours de formation LicenceMaster PICS, liant la physique et l'électronique avec ses applications, notamment dans le domaine de la photonique, a obtenu du réseau FIGURE [2] sa labellisation en Cursus de Master en Ingénierie (CMI) depuis 2013. Le lien entre la formation, la recherche et l'innovation est une des composantes fondamentales des CMI. Elle nécessite la mise en place d'outils pédagogiques en pointe avec les nouvelles technologies et adaptés à la formation en 5 ans des étudiants aux métiers d'ingénieur et/ou de la recherche. Dans les compétences visées par le cursus, on trouve notamment l'instrumentation et la métrologie optique, ainsi que la théorie de l'information et le traitement avancé des images. Outre les aspects théoriques de l'optique physique, il est incontournable de les aborder du point de vue de l'expérience et de les confronter à des modélisations numériques. Les fondamentaux du traitement du signal associés à toute la chaine d'acquisition des données menant à un format numérique ou au contraire à une conversion analogique sont nécessaires pour réaliser des mesures reproductibles et précises.
A travers un dispositif expérimental pédagogique d'holographie numérique dynamique, réalisé par des étudiants, il est proposé d'aborder tous ces aspects. Ainsi, cette approche permet une mesure de différence de trajets optiques atteignant des résolutions avoisinants la dizaine de nanomètres. Cette mesure correspond à la dimension en $\mathrm{z}$ (ou niveaux de gris de l'image). Les résolutions spatiales dans le plan (x,y) sont de l'ordre de la dizaine de micromètre. Les applications visées sont l'imagerie quantitative à contraste de phase de microcomposants (de type MEMS) ou des cellules biologiques.

L'holographie numérique [3-5] consiste à enregistrer sur une caméra 2D CCD ou CMOS un hologramme constitué de la superposition cohérente d'une onde de référence avec onde diffractée par un objet. La restitution de l'image s'effectue par le calcul numérique de la distribution du champ lumineux en intensité et en phase propagée sur la distance caméra-objet.

L'holographie numérique dynamique consiste à ajouter dans le montage un composant adressable numériquement, un modulateur spatial de lumière (SLM pour Spatial Light Modulator). Il se comporte comme un afficheur matriciel constitué de pixels indépendants (approximativement 1 Mpixels) capables d'effectuer une modulation en phase et/ou en intensité d'un champ lumineux incident. Il donne ainsi une plus grande flexibilité au montage. Des objets de phase sont générés grâce au SLM, et les aberrations du montage peuvent être compensées. L'optique dynamique connait un formidable essor ces dernières années grâce à l'émergence de 
ces composant adressables bidimensionnelles SLM souvent à base de cristaux liquides déposés sur silicium (LCOS : Liquid Crystal On Silicon). Citons les travaux les plus significatifs en optique dynamique de ces dernières années, les pinces optiques, l'affichage holographique, les procédés d'usinage laser à impulsions ultracourtes, la correction de front d'onde pour l'imagerie ou enfin l'optique adaptative [6-8].

\section{CONTEXTE ET ENJEUX PEDAGO- \\ GIQUES}

Le dispositif expérimental proposé aborde d'une part les concepts d'optique physique comme l'interférométrie, la diffraction en théorie scalaire, l'optique de Fourier et l'holographie, et d'autre part l'instrumentation optique/optoélectronique. Ce dernier aspect garantit l'acquisition et le traitement fiables des données pour élaborer une mesure optique avec une haute précision (nanométrique). Ces concepts sont en lien avec les Unités d'Enseignement (UE) de la licence de physique $3^{\text {ème }}$ année de l'UFR ST (UE Électromagnétisme et Optique Ondulatoire (16h CM/12h TD), UE Laser et Optique de Fourier (20h CM/TD)), et ont pu être approfondis à l'occasion d'un projet de fin d'études de licence, proposé à un binôme d'étudiants en 2014 (à raison d'une demijournée de travail expérimental sur un semestre). Plus tard dans le cursus, en master $2^{\text {ème }}$ année, une UE intitulée Mesure Optique et Vision [1] a pour objectif de former les étudiants à la métrologie optique bidimensionnelle à travers des méthodes de lumière structurée et/ou des approches interférométriques comme l'holographie ou l'interférométrie à décalage de phase (16h CM/TD intégrer, $10 \mathrm{~h} \mathrm{TP}$ sur les modèles numériques en codage Matlab, puis $8 \mathrm{~h}$ en travail pratique en labo). Les concepts d'optique physique sont à nouveau étudiés mais avec l'exigence supplémentaire d'y associer la théorie de l'information et du traitement de l'image numérique, la modélisation numérique de la propagation du champ lumineux diffracté en espace libre. L'instrumentation et les dispositifs optiques employés, et les limitations que cela implique, sont de même considérés.

Le travail de projet de fin d'étude de licence (équivalent à $120 \mathrm{~h}$ de travail étudiant) complète l'enseignement de façon à s'assurer de la formation effective des étudiants aux savoirs faire visés par les modules du diplôme de la licence mais permet aussi une ouverture à la recherche réalisée au sein du département d'Optique de l'institut FEMTO-ST. Le stage de Master (5 mois minimum) est quant à lui une immersion complète dans l'exigence d'un travail demandé à un jeune chercheur. Ce dernier doit faire preuve d'autonomie, de prise d'initiative et de créativité dans son travail, tout en démontrant sa capacité à mobiliser tous ses savoirs et ses savoir-faire acquis en 5 ans de formation Licence-Master.

Les principales difficultés rencontrées par les étudiants concernent leurs capacités à intégrer un certain nombre de concepts (théorique, numérique et pratique) sur une expérience unique, et sont très variables en fonction de leur niveau d'étude. A un niveau licence, un premier travail insiste plus sur l'élaboration de l'interféromètre et sur l'acquisition des hologrammes sur la caméra, mais aussi traite de la mise en forme et le filtrage spatial du faisceau laser incident. Il s'agit aussi de discuter des limites du montage. Un autre travail concerne l'étude, le fonctionnement et les propriétés du modulateur de phase spatial (SLM), comme par exemple, la calibration de l'état de polarisation rectiligne du faisceau incident pour obtenir une modulation de phase spatiale optimale. A un niveau master, un niveau d'autonomie supérieur est attendu sur l'expérience, le travail est très orienté vers la modélisation, le calcul numérique et les restitutions des hologrammes, avec une comparaison systématique avec les résultats de l'expérience. Ce travail mène ensuite l'étudiant à déterminer les paramètres physiques qui fixent la sensibilité et les résolutions accessibles dans le cadre de l'expérience.

Le montage d'holographie dynamique présenté peut être abordé et étudié à différents niveau de la formation (de la licence au master), et couvre le champ vaste de l'optique physique, de la théorie de l'information de la métrologie optique et de ses applications. A l'origine, ce banc expérimental a été réalisé à l'issus de travaux menés à l'institut FEMTO-ST concernant la mise en forme spatiale de faisceaux laser à impulsions ultracourtes par un SLM pour le micro-nano-usinage de matériaux [8$11]$.

\section{THEMES ABORDEES}

\subsection{Optique physique et expérimentation}

L'optique physique constitue une grosse partie du programme en licence $3^{\text {ème }}$ année, et s'étend de l'électromagnétisme à l'optique ondulatoire incluant l'instrumentation. Ceci a pour conséquence d'être parfois mal compris par les étudiants qui assimilent difficilement la variété des concepts étudiés ainsi que la multitude des expériences associées. C'est pourquoi des thèmes comme l'holographie ou la possibilité d'accéder à l'information 3D d'une image donne un sens et une motivation aux étudiants autour d'une grande variété des thèmes abordés : l'imagerie et l'instrumentation optique, la polarisation, la notion de cohérence d'une source, l'interférométrie et la théorie de la diffraction scalaire.

Un aspect tout aussi essentiel pour la formation de nos étudiants repose sur sa capacité à réaliser des expériences à partir de concepts théoriques. Il s'agit aussi d'interpréter les résultats obtenus et de les confronter aux résultats de simulations numériques (à niveau master au moins).

\subsection{Acquisition d'une image numérique et métro- logie}

L'expérimentation nécessite maintenant de passer d'un domaine purement analogique à un domaine numérique. L'optique et l'imagerie n'échappent pas à la règle. Une expérience d'interférométrie classique, par exemple, peut s'effectuer à l'aide d'une caméra CCD ou CMOS, 
afin d'enregistrer puis de traiter les figures d'interférences observées.

Il est important toutefois, de bien sensibiliser les étudiants sur l'effet du passage d'un signal analogique à un signal numérique. Il s'agit aussi de discuter des limites et des avantages que cela induit. Même si pour le grand public l'acquisition d'une image par une caméra se transfert facilement sur un des « écrans » du domicile, la théorie du signal et des images, de leurs acquisitions et de leurs traitements, de par leurs caractéristiques 2D $3 \mathrm{D}$ constituent une discipline à part entière. Pour des étudiants poursuivant un cursus de physique appliquée, il est primordial pour eux d'être en capacité d'étudier et d'intégrer les effets et les limitations de l'échantillonnage et de la quantification d'une image.

Pour ce faire, l'acquisition d'une image numérique dépend des caractéristiques physiques du capteur (optique, taille et nombre des pixels, niveau de quantification etc...), et son format au final repose sur une distribution d'intensité lumineuse pixélisée et codée en niveaux de gris. Si une mesure quantitative est requise, par exemple avoir accès à la topographie $3 \mathrm{D}$ de la surface d'un échantillon, c'est l'instrumentation optique et le procédé métrologique qui apporteront la mesure. Pour réaliser une topographie d'une surface, il s'agit par exemple d'associer à chaque niveau de gris de chaque pixel de l'image enregistrée, une échelle en unité de longueur. Les méthodes interférométriques optiques donnent accès à des mesures sans contact de la topographie d'échantillon avec des résolutions avoisinant le nanomètre. L'holographie numérique plus particulièrement est capable de réaliser de telles mesures sans systèmes imageurs, si l'échantillon est de dimension comparable à la taille du capteur de la caméra utilisée (quelques millimètres de côté). Faire ainsi travailler les étudiants sur l'holographie permet d'aborder de façon très complète un ensemble de concepts des plus fondamentaux aux plus appliqués.

\subsection{Holographie numérique}

L'holographie numérique consiste à enregistrer un hologramme directement sur un capteur matriciel de type CCD ou CMOS. La restitution de l'image n'est plus assurée par une voie optique, mais par calcul numérique. La reconstruction de l'image est synthétique et repose sur des algorithmes simulant la diffraction de l'onde de référence par l'hologramme et la propagation de ce champ diffracté du plan de l'hologramme au plan image. Ses avantages sont certains et idéaux pour des applications dans des environnements industriels : le développement dans les bains chimiques est supprimé, une sensibilité grande et une rapidité de l'acquisition, le stockage est facile et l'accès aux données pour des traitements numériques est direct.

L'holographie de Fresnel consiste à enregistrer directement sur une caméra (sans système imageur et à distance finie), l'interférence du champ diffracté par la surface éclairée de l'objet avec une onde de référence.
L'holographie de Fraunhofer constitue un cas particulier, où la diffraction du champ objet est captée à distance infinie (ou en plaçant l'objet au foyer d'une lentille). Les techniques d'interférométrie holographique, déjà beaucoup utilisées en holographie classique, sont bien sûr applicables directement avec les supports à matériaux solides (CCD, CMOS).

Néanmoins, la principale limite de l'holographie numérique provient de la basse résolution spatiale des capteurs CCD ou CMOS. Leur rôle est en effet d'enregistrer les micro-interférences constituant l'hologramme. Si $\theta$ représente l'angle entre les faisceaux de référence et objet, la période de ces franges $\delta$ en intensité est donnée par la relation suivante :

$$
\delta=\frac{\lambda}{2 \sin \theta / 2}
$$

Pour un capteur CMOS possédant des pixels espacés d'une distance $\Delta$, le théorème de Shannon stipule que deux pixels au moins par période de frange $(2 \Delta<\delta)$ sont nécessaires pour assurer un échantillonnage correct, ce qui se traduit par la relation suivante dans les approximations des petits angles :

$$
\theta<\frac{\lambda}{2 \Delta}
$$

Ces conditions compliquent la géométrie des montages expérimentaux car cela implique de travailler avec des faisceaux quasi-parallèles, c'est-à-dire avec des objets de petites tailles disposés à de grandes distances du capteur. Malgré de telles restrictions, l'holographie numérique trouve un panel d'applications variées, surtout dans la métrologie sans contact et l'imagerie à contraste de phase quantitative d'objet biologique ou de micro-objets.

\subsubsection{Restitution numérique}

L'idée d'un traitement numérique des hologrammes est en réalité assez ancienne. En 1966, Brown et Lohmann décrivirent le principe des premiers hologrammes binaires générés par ordinateur ("Computed Generated Holograms" ou CGH). Cette approche diffère de l'holographie numérique, car il s'agit de calculer la répartition d'intensité générée dans le plan de l'hologramme, par l'interférence d'un front d'onde objet simulé avec une onde de référence simulée également. Ensuite, son image est reconstruite par une voie optique classique.

Ainsi, le calcul de fronts d'ondes objets idéaux ou parfaits est envisageable et permet de réaliser, par exemple, des composants optiques de type microlentilles (HOE "Holographic Optical Elements") sans procédé de fabrication, souvent coûteux et délicats. Les applications des CGH sont multiples. Elles concernent les filtres spatiaux, les composants optiques, l'interconnectique, les mémoires holographiques et le stockage de données, la génération artificielle d'images...

Les algorithmes développés pour les CGH, simulant la propagation des fronts d'ondes et basés sur l'intégrale de diffraction de Fresnel-Kirchoff, ont été repris en holographie numérique pour la reconstruction numérique de l'image. Yaroslavskii et Merzlyakov (1980) 
[12] ont traité la possibilité de cette restitution synthétique des fronts d'ondes à partir d'hologrammes échantillonnés. Plus tard, Schnars (1994) [13] a continué sur cette voie et publié les premiers résultats expérimentaux sur l'holographie numérique de Fresnel hors axe.

L'holographie est basée sur la nature ondulatoire de la lumière. Une description de la propagation des ondes lumineuses passe par une connaissance a priori des effets de la diffraction. La restitution synthétique repose sur la théorie scalaire de la diffraction. Les deux principales approches sont la diffraction de Fresnel ou l'approche par spectre angulaire d'ondes planes [9, 14].

\subsection{Optique dynamique}

Dès les années 50, en imagerie pour l'astronomie, une instrumentation pour les télescopes terrestres s'est développée pour corriger les distorsions des fronts d'ondes traversant l'atmosphère. Le terme alors employé était celui d'optique active, puisque cela consiste à recourir à des miroirs déformables pour compenser les déformations des fronts d'ondes et les aberrations tout au long de la propagation de l'onde.

Depuis plus d'une vingtaine d'année, l'optique adaptative a émergé car elle est capable d'effectuer des corrections de front d'ondes en temps réel (la centaine de $\mathrm{Hz}$ ). Ces dispositifs reposent essentiellement sur des approches géométriques, avec des analyseurs de front d'ondes de type Shack Hartmann.

Plus récemment, le début des années 2000 correspond à l'arrivée de nouvelles générations d'analyseur de front d'onde basées sur des dispositifs à cristaux liquides pixélisés, les SLM. Ils sont capables d'effectuer une modulation d'onde incidente en amplitude et/ou en phase. La modulation phase atteint maintenant une valeur de $2 \pi$ par pixel et s'obtient à partir d'une image numérique (format VGA ou supérieure suivant les modèles et codées en $8 \mathrm{bit} / 256$ niveaux de gris).

Comme les SLM autorisent une plus grande flexibilité dans l'adressage des fonctions optiques accessibles dans des expériences d'optique, y compris interférométrique, on parle maintenant d'optique dynamique (en y incluant l'optique adaptative avec des techniques de corrections de fronts d'ondes en temps réel avec des approches interférométriques).

L'optique dynamique trouve des applications non seulement en astronomie, mais aussi en imagerie médicale, en procédé d'usinage laser et dans les télécommunications.

\section{MONTAGE EXPERIMENTAL}

\subsection{Schéma du montage}

Le montage expérimental (fig. 1) est volontairement simple, puisqu'il est constitué d'une source laser (HeNe $\lambda=632,8 \mathrm{~nm}$ ), d'un système optique d'élargissement du faisceau avec un trou de filtrage spatial, et enfin d'un interféromètre de Michelson. Ce dernier est constitué d'un miroir sur le bras du faisceau de référence, et le SLM est placé sur le bras du faisceau objet. Ainsi, la fonction première du SLM est de simuler un objet de phase dynamique (car ré-adressable ou modifiable en temps réel). Sa deuxième fonction étant d'effectuer une

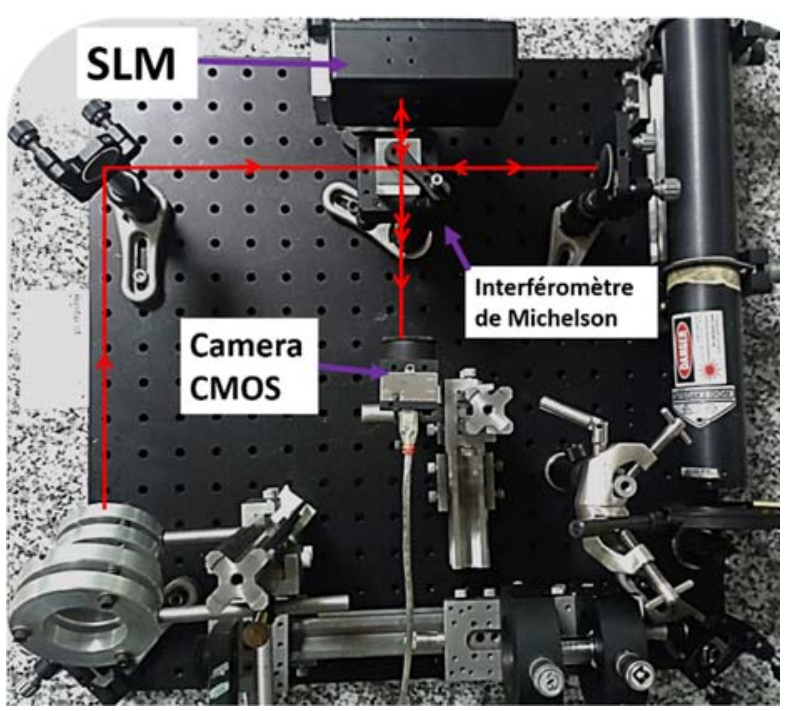

Fig. 1 Montage d'holographie numérique dynamique

correction des distorsions et des aberrations éventuelles du montage.

Le SLM utilisé est de type SLM-LCOS X10468 (Hamamatsu, 792x600 pixels de $20 \mu \mathrm{m}$ ). Le faisceau incident est polarisé linéairement selon une des lignes neutres du SLM afin d'effectuer une modulation de phase de l'onde.

Un cube séparateur 50/50 réalise la séparation puis la superposition cohérente de l'onde objet et de l'onde de référence en introduisant un angle $\theta$ (équation 2) pour être en holographie de Fresnel hors axe.

Les hologrammes numériques sont enregistrés sur une caméra CMOS $(5 \mathrm{Mp}$ monochrome $\mu$ eye 1920x2560 pixels de 2,2 $\mu \mathrm{m}$ ) placée à une distance d d'environ $15 \mathrm{~cm}$ du SLM (distance objet-hologramme). Aucune lentille n'est utilisée pour réaliser l'image de

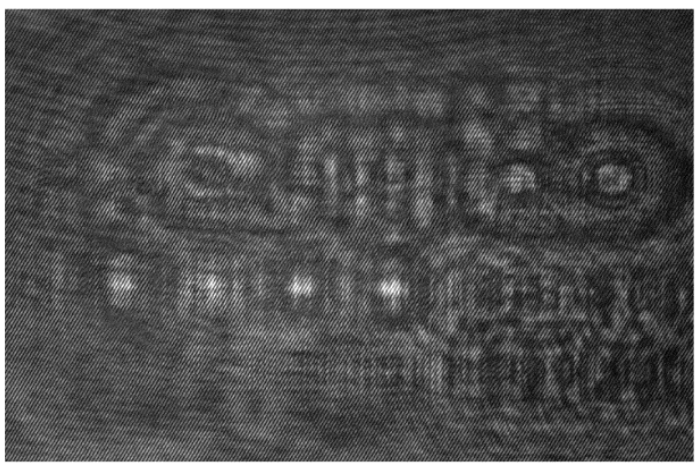

Fig. 2 Hologramme numérique d'un objet de phase généré via le SLM, la dimension de l'hologramme correspond à celle de la caméra CMOS : 4,2x5.6 mm²

l'objet de phase. Elle sera restituée en intensité et en phase par restitution numérique. 
La surface photosensible de la caméra vaut $4,2 \times 5,6 \mathrm{~mm}^{2}$, elle est donc plus réduite que la surface du

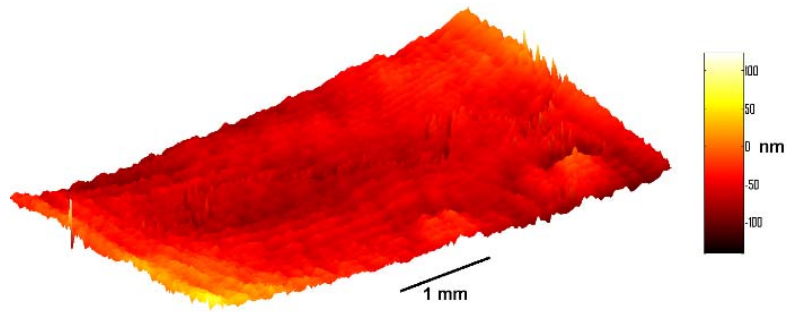

Fig. 3 Distribution de phase $3 D$ non corrigée d'un objet de phase plan et restituée à une distance $d=15,3 \mathrm{~cm}$. Planéité mesurée en différence de trajet optique : $37,2 \mathrm{~nm} \mathrm{rms}$

SLM $\left(15,8 \times 12 \mathrm{~mm}^{2}\right)$. Le champ d'observation est celui de la caméra puisque l'interféromètre est illuminé en faisceau parallèle (assimilé à des ondes planes).

\section{RESULTATS ET MESURES}

La Figure 2 montre un hologramme numérique enregistré sur la caméra CMOS d'après le montage de la Figure 1. L'objet est généré via le SLM à partir d'une image numérique codée en 256 niveaux de gris ( 8 bit).

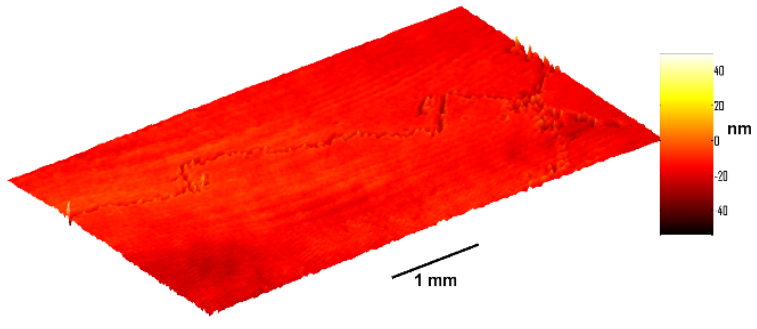

Fig. 4 Distribution de phase 3D corrigée d'un objet de phase plan et restituée à une distance $d=15,3 \mathrm{~cm}$. Planéité mesurée en différence de trajet optique : 14,6 $\mathrm{nm}$ rms

Une première étape consiste à mesurer la qualité du front d'onde correspondant à la différence du trajet optique entre les bras de référence et objet, lorsque l'objet est un plan de phase pur (un niveau de gris constant sur le SLM). La Figure 3, montre la cartographie de phase 3D obtenue par restitution calculée de l'hologtamme numérique (algorithme programmé à partir du spectre d'ondes planes, non détaillé ici). La planéité ramenée en différence de trajet optique est mesurée à $37 \mathrm{~nm}$ RMS. On observe clairement les distorsions du front d'onde, qui restent notables.

Si on compare cette valeur à la planéité donnée par le constructeur du SLM, qui est de $8 \mathrm{~nm}$ RMS à la longueur d'onde utilisée, notre mesure est nettement détériorée par les autres éléments du montage (leurs défauts - miroir et cube séparateur - sont aussi pris en compte dans la mesure et s'ajoutent à ceux du SLM).
Une correction du front d'onde est alors appliquée en soustrayant deux plans de phase restitués issues de deux différentes acquisitions. La Figure 4 montre alors le résultat de cette correction, avec une planéité en différence de trajet optique obtenue à $14,6 \mathrm{~nm}$. Ce résul-

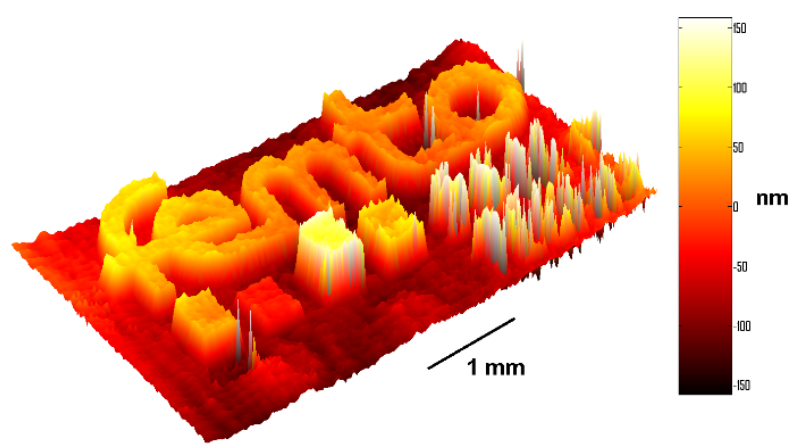

Fig. 6 Distribution de phase 3D non corrigée restituée à une distance $d=15,3 \mathrm{~cm}$

tat est comparable aux valeurs données par le constructeur et on montre ici que les effets des éléments supplémentaires du montage sont en partie compensés.

La Fig. 5 montre l'image du logo de FEMTO-ST restituée en intensité à partir d'un hologramme numérique enregistré sur la caméra. Le logo est appliqué en niveaux de gris comme un objet de phase multiniveaux sur le SLM. Aucune mesure de différence de trajet optique n'est accessible sur cette figure. En effet,

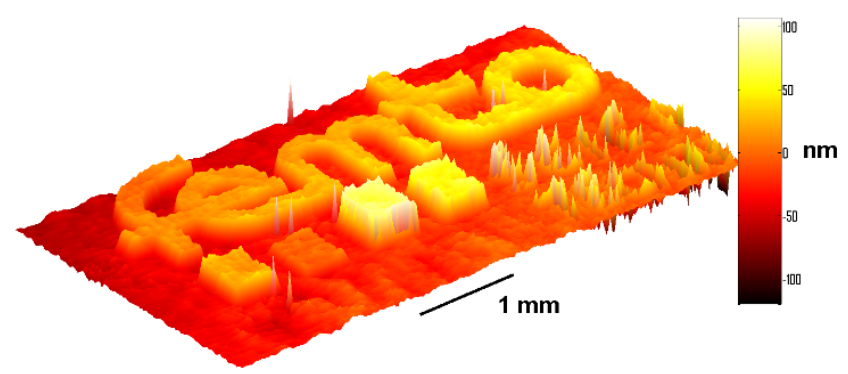

Fig. 5 Distribution de phase $3 D$ corrigée restituée à une distance $d=15,3 \mathrm{~cm}$

les niveaux de gris sont donnés en unité arbitraire. Elle permet toutefois d'ajuster la distance de focalisation numérique $d$ de restitution. Elle montre que le logo FEMTO n'est visible que par ses contours témoigant d'un objet de phase pur, ce qui n'est pas le cas pour le bout de texte "Sciences et Techniques ». En effet, un objet de phase pure correspond à une profondeur de modulation de $2 \pi$ (liée à une valeur de niveaux de gris en fonction de la longueur d'onde, pour le SLM cette valeur est donnée à 118 à $\lambda=633 \mathrm{~nm}$ sur les 256 niveaux du codage 8bit). Le logo FEMTO-ST est codé en niveaux de gris à différentes profondeurs de modulation (les 4 carrés et le texte "Sciences et Techniques »), introduisant une modulation d'amplitude supplémentaire dans l'image restituée de la Fig. 5. 


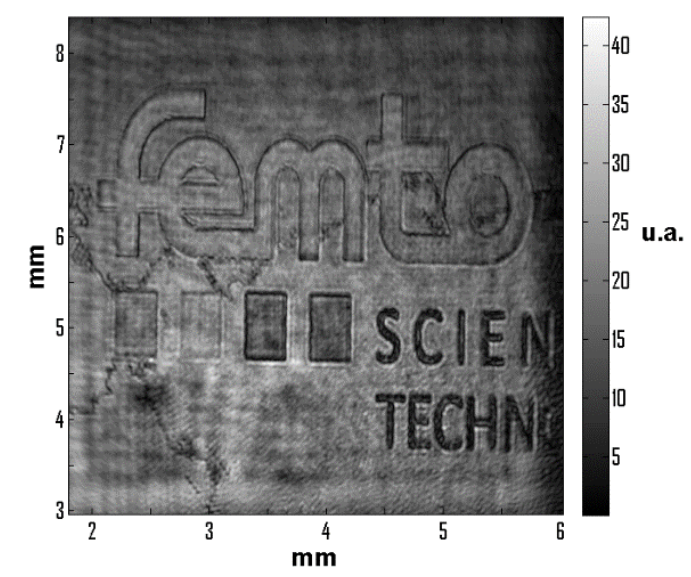

Figure 7 Distribution d'intensité restituée à $d=15.3 \mathrm{~cm}$ d'un hologramme numérique généré à partir du logo de l'institut FEMTO-ST en phase appliqué au SLM

Les figures 6 et 7 représentent l'image du logo restituées en phase 3D sans et avec correction du front d'onde. La hauteur des motifs en différence de trajets optiques varie de 20 à $100 \mathrm{~nm}$, et correspond à une topographie équivalente de l'objet "synthétique » généré par le SLM. Dans le plan de l'hologramme, les résolutions latérales sont à ramener à la dimension des NxM pixels de la caméra $(\Delta=2,2 \mu \mathrm{m})$. En revanche, dans le plan de l'image restituée, les résolutions latérales $(\Delta x$, $\Delta y$ ) dépendent de la tache de diffraction sur la distance de propagation d. Elles diffèrent en $\mathrm{x}$ et $\mathrm{y}$ car le nombre de pixels verticaux $(\mathrm{N}=1920)$ et horizontaux $(\mathrm{M}=2560)$ sont différents. Ainsi, dans l'approximation paraxiale, on trouve que :

$$
\Delta x=\frac{\lambda d}{N \Delta}, \Delta y=\frac{\lambda d}{M \Delta}
$$

On obtient ainsi des résolutions latérales d'une vingtaine de micromètres $((\Delta \mathrm{x}=23 \mu \mathrm{m}, \Delta \mathrm{y}=17 \mu \mathrm{m})$ dans le plan de l'image restituée.

\section{6}

\section{CONCLUSIONS, PERSPECTIVES}

Le travail proposé s'appuie d'une part sur des réalisations expérimentales et numériques d'étudiants en licence $3^{\text {ème }}$ année de physique et en master $2^{2 \text { ème année }}$ PICS de l'UFC, mais aussi sur des travaux de recherche menés à l'institut FEMTO-ST pour le contrôle actif de faisceaux lumineux par SLM pour des applications à l'usinage laser à impulsions ultracourtes de divers matériaux (transparents ou non, verre, métaux, semi-conducteurs...). Même si le SLM utilisé dans les présents travaux représente un investissement lourd $(>15 \mathrm{k} €$ ), et est plus en lien avec une activité de recherche, il existe des SLM à environ $1000 €$, qui sont compatibles avec les expériences d'holographie dynamique comme ceux proposés par Cambridge Correlator [14].

La variété des disciplines, des concepts et des thèmes abordés à travers la mise en place d'une expérience d'holographie numérique dynamique participe à un approfondissement des savoirs et des savoir-faire attendus de futurs diplômés, et rentre pleinement dans les objectifs de spécialisation visés par le label CMI PICS.

\section{Remerciements}

Ce travail a bénéficié d'une aide de l'Etat gérée par l'ANR au titre du programme d'Investissement d'avenir LABEX ACTION portant la référence : ANR-11-LABX-0001-01) et de la région Franche-Comté.

\section{Bibliographie}

[1] Université de Franche-Comté, CMI PICS (Photonique, Micro-nanotechnologies et Temps-fréquence), UFR Sciences et Technique, http://sciences.univfcomte.fr/pages/fr/menu3795/formations/cmi-cursus-demaster-en-ingenierie-15940.html et le site du master PICS : http://sdm.univ-fcomte.fr

[2] http://www.reseau-figure.fr/.

[3] T.Kreis, "Handbook of Holographic Interferometry, Optical and Digital Methods", Wiley-VCH, Berlin (2004)

[4] T.-C. Poon, J.-P. Liu "Introduction to modern digital holography with matlab", Cambridge University Press, New York, 2014.

[5] J.-C. Li, P. Picart, " Holographie numérique : principe, algorithmes et applications », Lavoisier, Paris (2012).

[6] S. Zwick, T. Haist, M. Warber, and W. Osten, "Dynamic holography using pixelated light modulators," Appl. Opt. 49, F47-F58 (2010)

[6] M. Reicherter, T. Haist, E. U. Wagemann, and H. J. Tiziani, "Optical particle trapping with computer-generated holograms written on a liquid-crystal display," Opt. Lett. 24, 608-610 (1999)

[7] MK Bhuyan, F Courvoisier, PA Lacourt, M Jacquot, R Salut, L Furfaro, JM Dudley, "High aspect ratio nanochannel machining using single shot femtosecond Bessel beams," Appl. Phys. Lett., 97, 081102, (2010)

[8] L. Froehly, M. Jacquot, P. A. Lacourt, J. M. Dudley, and F. Courvoisier, "Spatiotemporal structure of femtosecond Bessel beams from spatial light modulators," J. Opt. Soc. Am. A 31, 790-793 (2014)

[9] F. Courvoisier, A. Mathis, L. Froehly, R. Giust, L. Furfaro, P. A. Lacourt, M. Jacquot, and J. M. Dudley, "Sending femtosecond pulses in circles: highly nonparaxial accelerating beams," Opt. Lett. 37, 1736-1738 (2012)

[10] A. Mathis, F. Courvoisier, R. Giust, L. Furfaro, M. Jacquot, L. Froehly, and J. M. Dudley, "Arbitrary nonparaxial accelerating periodic beams and spherical shaping of light," Opt. Lett. 38, 2218-2220 (2013)

[11] L. P. Yaroslavskii, N. S. Merzlyakov, Methods of Digital Holography, Consultants Bureau, New York, 1980

[12] U. Schnars and W. Jüptner, "Direct recording of holograms by a CCD target and numerical reconstruction," Appl. Opt. 33, 179-181 (1994)

[13] P. Sandoz and M. Jacquot, "Lensless vision system for inplane positioning of a patterned plate with subpixel resolution," J. Opt. Soc. Am. A 28, 2494-2500 (2011)

[14] R. Bowman1, V. D’Ambrosio, E. Rubino, O. Jedrkiewicz, P. Di Trapani, M.J. Padgett "Optimisation of a lowcost SLM for diffraction efficiency and ghost order suppression.” Eur. Phys. J. Special Topics 199, 149-158 (2011) 